Supporting Information

\title{
Confined Reduced Graphene Oxides as a Platform for DNA Sensing in Solutions Crowded with Biomolecules
}

Yuuki Hata, ${ }^{\dagger}$ Toshiki Sawada, ${ }^{\dagger+}$ and Takeshi Serizawa ${ }^{* \dagger}$

†Department of Chemical Science and Engineering, School of Materials and Chemical Technology, Tokyo Institute of Technology, 2-12-1 Ookayama, Meguro-ku, Tokyo 152-8550, Japan.

†Precursory Research for Embryonic Science and Technology (PRESTO), Japan Science and Technology Agency (JST), 4-1-8 Honcho, Kawaguchi-shi, Saitama 332-0012, Japan.

*E-mail: serizawa@polymer.titech.ac.jp 
Figure S1. Synthetic reaction of cellulose oligomers.

Figure S2. UV-Vis absorption spectra of the (r)GOs.

Figure S3. Raman spectra of the (r)GOs.

Figure S4. ${ }^{1} \mathrm{H}$ NMR spectrum of the cellulose oligomers.

Figure S5. ATR-FTIR absorption spectrum of the products.

Figure S6. SEM image of the xerogels.

Figure S7. AFM image of the cellulose oligomer nanoribbons. S5

Figure S8. Optical microscopy images of the crushed gels. S6

Figure S9. Photographs of the crushed gel dispersions before and after centrifugation. S6

Figure S10. UV-Vis absorption spectra of the (r)GOs extracted from the hybrid gels prepared at 60, 45, or

$30{ }^{\circ} \mathrm{C}$.

Figure S11. Sensing in the presence of various concentrations of bovine serum albumin using the hybrid gels prepared at 60,45 , or $30{ }^{\circ} \mathrm{C}$.

Figure S12. Linear fitting of fluorescence intensity in the sensing of 0-20 nM T1.

Figure S13. Photographs of the crushed freeze-dried gel dispersions and the freeze-dried GO dispersions.

Figure S14. Sensing in the presence of serum using never-dried or freeze-dried hybrid gels. 


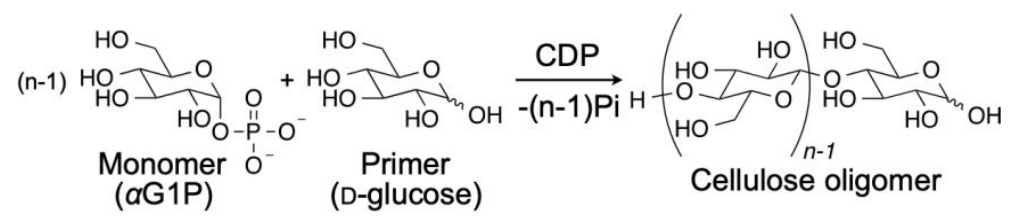

Figure S1. Synthetic reaction of cellulose oligomers via the cellodextrin phosphorylase-catalyzed enzymatic reaction.

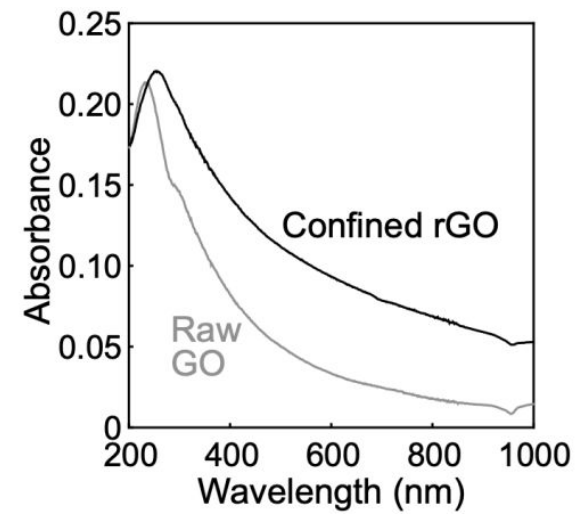

Figure S2. Ultraviolet-visible (UV-Vis) absorption spectra of the raw graphene oxides (GOs) and the confined reduced GOs (rGOs).

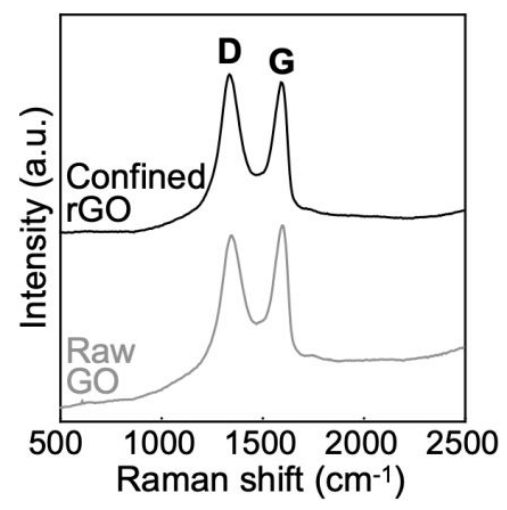

Figure S3. Raman spectra of the raw GOs and the confined rGOs. 


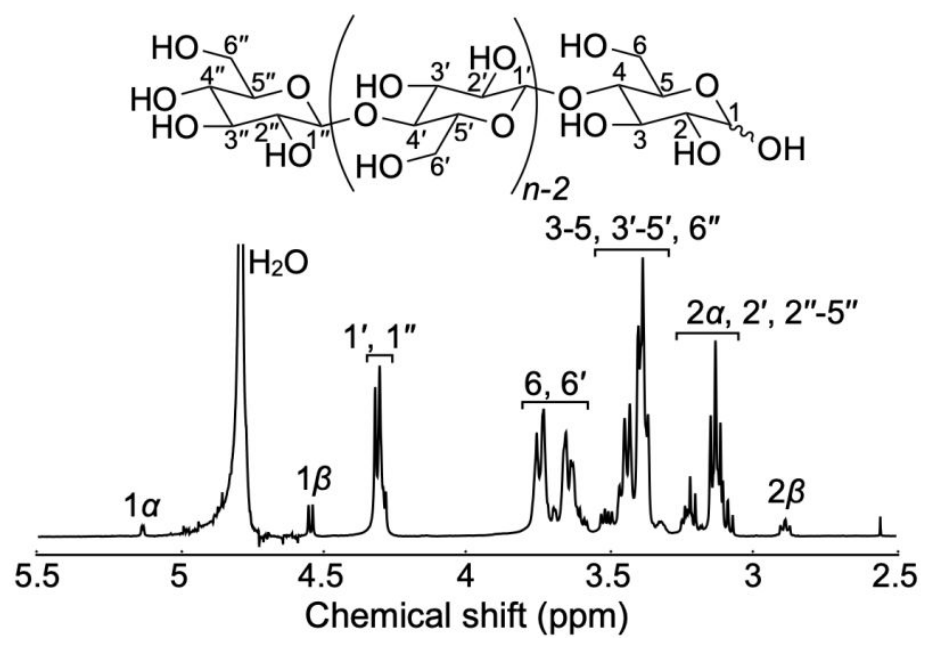

Figure S4. ${ }^{1} \mathrm{H}$ nuclear magnetic resonance (NMR) spectrum of the cellulose oligomers synthesized in GO dispersions.

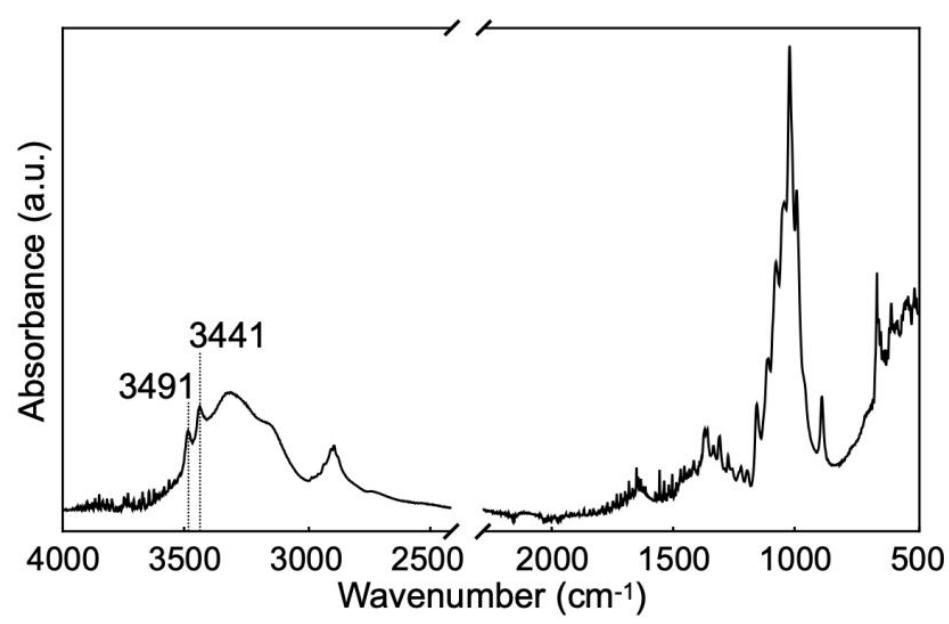

Figure S5. Attenuated total reflection-Fourier transform infrared (ATR-FTIR) absorption spectrum of the products. The spectrum showed two characteristic peaks for the intrachain hydrogen-bonded hydroxyl groups in the cellulose II allomorph at approximately 3,441 and $3,491 \mathrm{~cm}^{-1}$. 


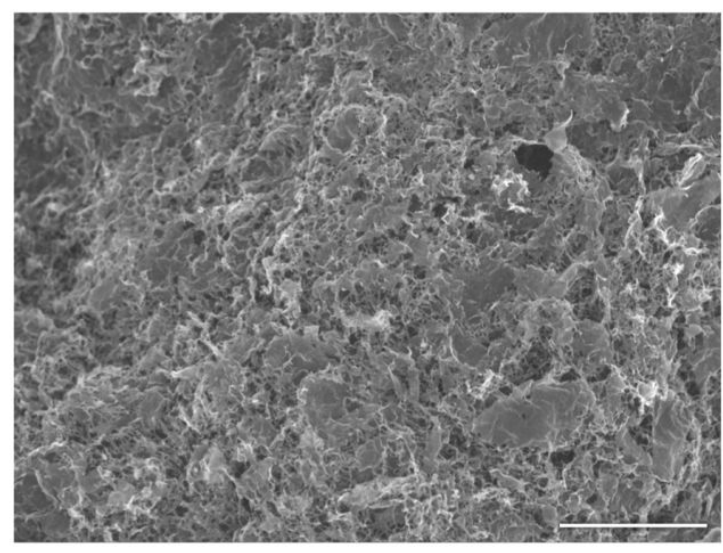

Figure S6. Scanning electron microscopy (SEM) image of the xerogels prepared from the hydrogels. The scale bar is $10 \mu \mathrm{m}$

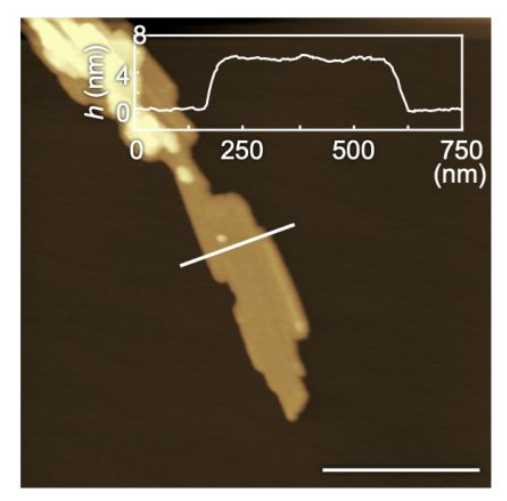

Figure S7. Atomic force microscopy (AFM) image of the mechanically crushed nanoribbons. Inset is a cross-section. The scale bar is $1 \mu \mathrm{m}$. 

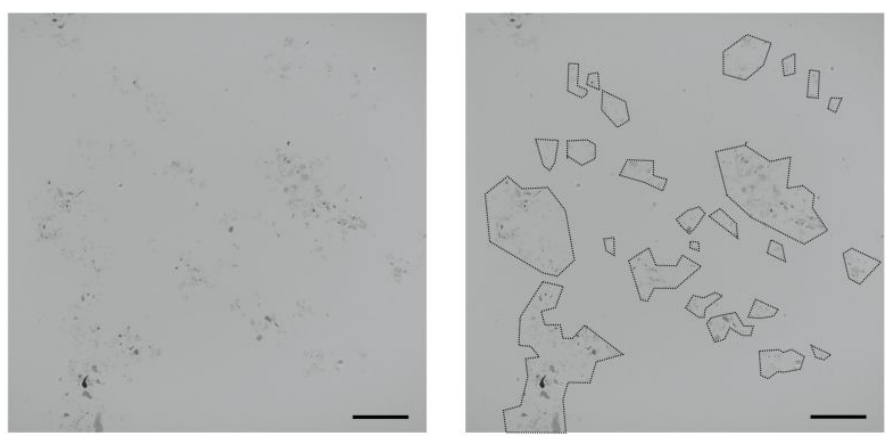

Figure S8. Optical microscopy images of the crushed gels. In the right image, the observed crushed gel particles were highlighted with dotted lines. The scale bars are $100 \mu \mathrm{m}$.
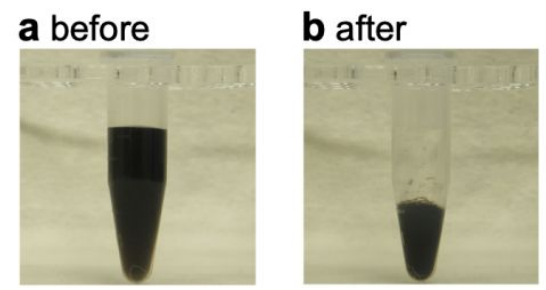

Figure S9. Photographs of the crushed never-dried gel dispersions $\left(0.25 \mathrm{mg} \mathrm{mL}^{-1} \mathrm{rGO}\right.$ ) (a) before and (b) after centrifugation $(20,400 \times g)$.
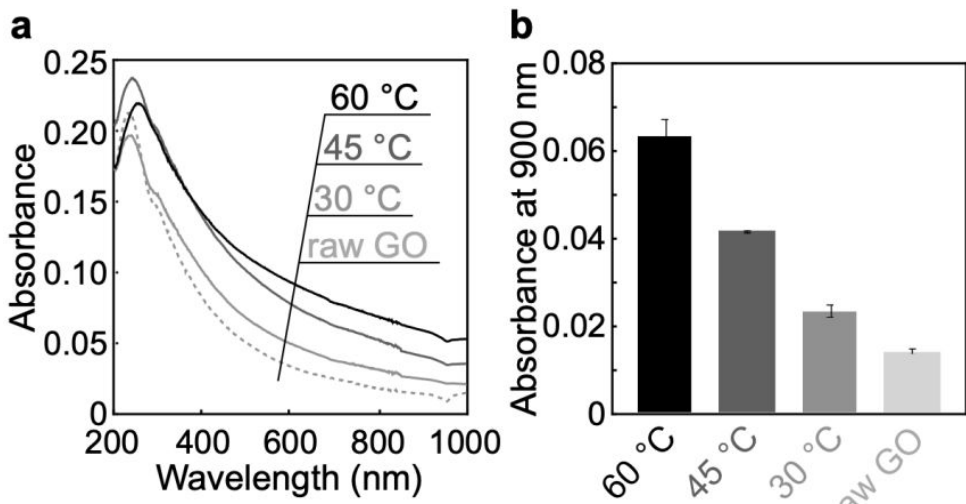

Figure S10. (a) UV-Vis absorption spectra and (b) absorbance at $900 \mathrm{~nm}$ of the raw GOs and of the (r)GOs extracted from the hybrid gels prepared at 60,45 , or $30^{\circ} \mathrm{C}$. The error bars in (b) represent the standard deviation of three individual trials. 

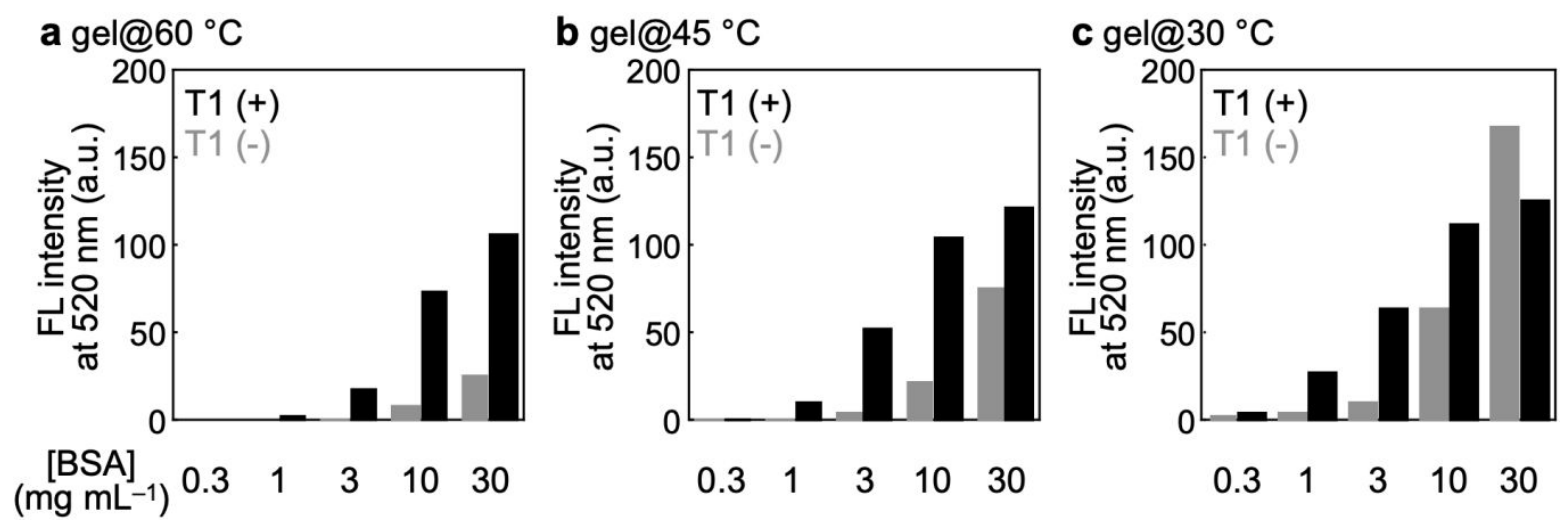

Figure S11. Sensing in the presence of various concentrations of bovine serum albumin using the hybrid gels prepared at (a) 60 , (b) 45 , or (c) $30^{\circ} \mathrm{C}$.

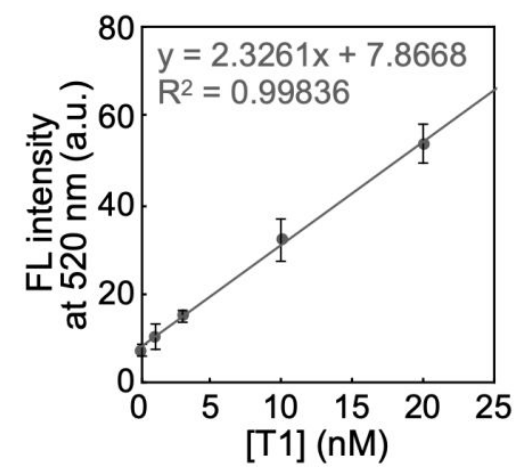

Figure S12. Linear fitting of fluorescence intensity at $520 \mathrm{~nm}$ in the sensing of $0-20 \mathrm{nM}$ T1. The error bars represent the standard deviation of three individual trials. 

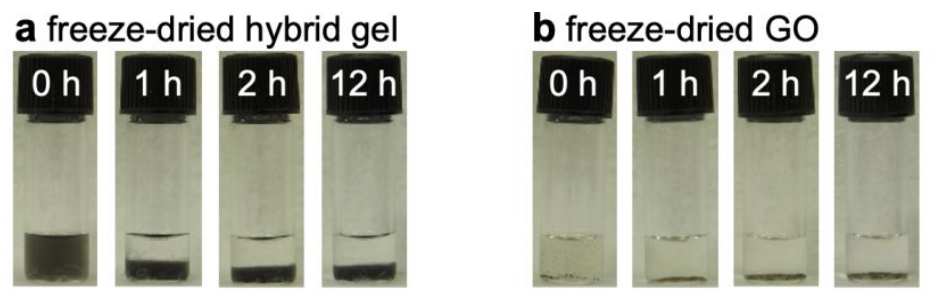

Figure S13. Photographs of (a) the crushed freeze-dried gel dispersions and (b) the freeze-dried GO dispersions after incubation for $0-12 \mathrm{~h}$.

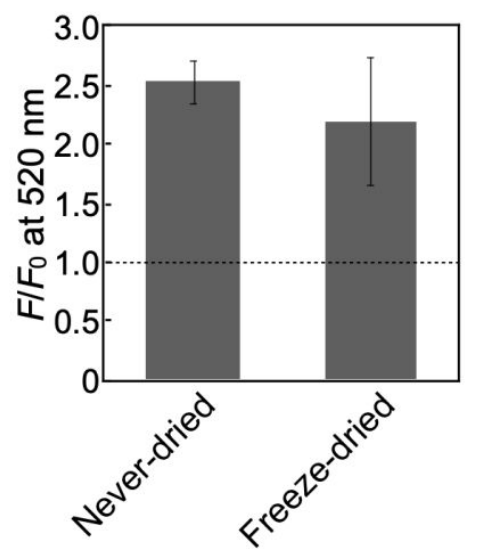

Figure S14. Signal-to-background ratios of the sensing system in the presence of serum using the neverdried or freeze-dried hybrid gels. The error bars represent the standard deviation of three individual trials. 